\title{
KRAS Codons 12 and 13 Mutation Analysis: A Comparative Study between Direct Sequencing and a New Sensitive Real-Time PCR Assay
}

\author{
Stefania Marzinotto, ${ }^{1}$ Francesca Sessa, ${ }^{2}$ Alessandra Franzoni, ${ }^{1}$ \\ Alessia Anselmi, ${ }^{2}$ Laura Rosa Gastaldo, ${ }^{2}$ Silvia Mason, ${ }^{2}$ Giuseppe Damante, ${ }^{1}$ \\ Carlo Alberto Beltrami, ${ }^{1}$ and Laura Mariuzzi ${ }^{1}$
}

${ }^{1}$ Dipartimento di Scienze Mediche e Biologiche, Università di Udine, 33100 Udine, Italy

${ }^{2}$ AB ANALITICA s.r.l., 35127 Padova, Italy

Correspondence should be addressed to Laura Mariuzzi, laura.mariuzzi@uniud.it

Received 29 July 2011; Revised 26 September 2011; Accepted 29 September 2011

Academic Editor: Luis Menéndez-Arias

Copyright (C) 2011 Stefania Marzinotto et al. This is an open access article distributed under the Creative Commons Attribution License, which permits unrestricted use, distribution, and reproduction in any medium, provided the original work is properly cited.

\begin{abstract}
KRAS somatic mutations are found in 30-40\% of colorectal cancer (CRC). Seven mutations in codons 12 and 13 of KRAS (95\% of the observed human mutations) preclude the efficacy of anti-EGFR therapy for the treatment of CRC. Assessment of KRAS mutational status has become a standard procedure in the management of patients with CRC. Technically, KRAS mutation testing can be performed with different methods, characterized by distinct sensitivities and specificities. The present study analyzed KRAS in 182 CRC histological samples by using direct sequencing and a new kit based on a Real-Time Sequence-Specific Primers-PCR technology. The kit allowed to recover as positive 17 samples that were negative or unclear by sequencing, with a recovery rate equal to $13.82 \%$. This study proposes a fast, sensitive, and high-throughput system to identify such seven described mutations of KRAS gene in CRC samples.
\end{abstract}

\section{Introduction}

Colorectal cancer (CRC) represents the third cause of death for malignant neoplasia in western countries. Even if in the last few years an increase in the incidence of CRC has been observed, a decreased mortality has been reported, mainly due to an adequate prevention, an early diagnosis, and the use of novel classes of therapeutic agents, which were developed through a larger and more exhaustive knowledge of the biology of cancer. The emerging picture is that CRC develops as a result of multiple sequential steps, with accrual of genetic alterations including mutations, gene amplification, and epigenetic changes [1-3]. Accumulating knowledge has led to the advent of new active chemotherapeutic agents and, especially, novel targeted drugs, which led to significant advances in outcome of CRC patients over the last decade [4-8].

The rationale of targeted therapies is to inhibit biological pathways and key molecules involved in tumour growth and progression $[9,10]$. Particularly, in the last few years, a therapeutic strategy was created to treat CRC through the administration of monoclonal antibodies (mAbs) against the Epithelial Growth Factor Receptor (EGFR) [11-14]. EGFR activation stimulates different intracellular signaling pathways that are tightly regulated by the presence and identity of the ligand, heterodimer composition, and the availability of phosphotyrosine-binding proteins. The two primary signaling pathways activated by EGFR include the RAS/RAF/ MEK/ERK and the PI3K/AKT axes [15-19]. Experimental pieces of evidence have shown that alterations depending on the EGFR receptor and/or on the activation of the effectors are involved in cancer initiation and progression, promoting mechanisms of resistance to apoptosis, tumor cell proliferation, survival, invasion, and angiogenesis. However, recent studies have demonstrated that the therapeutic effect of anti-EGFR mAbs is present in just $10-20 \%$ of the cases and that resistance to such a treatment is usually associated 
with the presence of mutations in the genes involved in EGFR signal transduction pathways $[17,20]$. The oncogene KRAS is indeed the most commonly mutated gene in different human cancers and its constitutive activation in CRC can bypass the EGFR-driven signaling cascade and impair the clinical efficacy of anti-EGFR mAb [20].

In biopsy samples, the volume fraction occupied by the tumor can be extremely heterogeneous. This somatic mosaicism is one of the main hurdles for the detection of point mutations. The amount of normal cells in the biopsy tissue "contaminates" the tumoral component, making harder the spotting of point mutations through direct sequencing. The fact that mutations are mainly found in heterozygosis further complicates the research of mutated alleles. Even if direct sequencing remains the most common technique to determine the exact kind of mutation, recent data have suggested the need to increase the sensitivity of the methods to detect mutations in KRAS for enhancing the prediction of resistance to cetuximab or panitumumab in CRC. There is urgency to establish widely accepted guidelines for KRAS testing, focused on defining the sensitivity threshold that is required for the accurate identification of nonresponder (NR) patients $[2,21-23]$. Although there is no real consensus, Sanger sequencing has been regarded as the gold standard methodology for KRAS mutation testing and is the most widely used technique. The major limitations of this method are the length of the process, the fairly low throughput, the expertise required by the technologists, and the high cost of sequencers. Furthermore, this methodology may have a low analytical sensitivity to detect mutations in samples with very low tumor content [24].

The aim of this study was to determine if REALQUALITY RI-KRAS MuST, a faster and more user-friendly assay, gives the same or more high-quality results as Sanger sequencing for KRAS mutation testing.

\section{Materials and Methods}

2.1. Tissue Specimens and Processing. Formalin-fixed and paraffin-embedded (FFPE) surgical biopsies from 182 CRC were obtained from the Department of Pathology of the University of Udine, Italy. Informed consent was requested from each patient. Five $\mu$ tissue sections were cut from paraffin-embedded tumour tissue blocks and stained with Haematoxylin and Eosin (H\&E) for histopathological examination. For DNA isolation, five sections, $10 \mu$ thick, were used. Deparaffination of the sections was performed, and, using the $\mathrm{H} \& \mathrm{E}$ section as reference, tumor tissue was macrodissected from the normal colonic epithelium and scraped off. Genomic DNA was extracted using QIAamp DNA mini kit (Qiagen, Milan, Italy). Briefly, each sample was incubated in $180 \mu \mathrm{L}$ of ATL buffer (tissue lysis buffer included in the kit) and $20 \mu \mathrm{L}$ of proteinase $\mathrm{K}$ at $56^{\circ}$ with shaking and left overnight to ensure that they were completely lysed. The sequencing fragment template was a 172-bp PCR fragment of the KRAS gene, generated with the primers KRAS F: 5' -AAGGCCTGCTGAAAATGACT-3' and KRAS R: 5'-CTGGTGCAGGACCATTCTTTGA-3' using 100-200 ng of genomic DNA. PCR conditions were as follows: initial denaturation for $5 \mathrm{~min}$ at $95^{\circ} \mathrm{C}$, denaturation for $30 \mathrm{~s}$ at $95^{\circ} \mathrm{C}$, annealing for $45 \mathrm{~s}$ at $56^{\circ} \mathrm{C}$, extension for $45 \mathrm{~s}$ at $72^{\circ} \mathrm{C}$, 35 cycles, final extension $72^{\circ} \mathrm{C}$ for $10 \mathrm{~min}$. High Pure PCR Product Purification Kit (Roche Diagnostics, Gmbh) cleaned up the PCR product. Cycle sequencing analysis of PCR fragments was done with BigDye Terminator (Applied Biosystems) system using reverse amplification primer. The reaction products were analyzed on an ABI PRISM 310 (Applied Biosystems).

All cases were analyzed twice with independent PCR reaction according to the Italian Society of Pathology-International Academy of Pathology (IAP) guidelines (November 2010).

The main goal of this work was to compare the performance of REALQUALITY RI-KRAS MuST (AB ANALITICA, Padua, Italy) to the results obtained with direct sequencing. Because of the different levels of sensitivity between the two systems, it was necessary to use other systems in order to validate the results obtained with REALQUALITY RI-KRAS MuST. For this purpose, mutation analysis was performed also with Restriction Fragment Length Polymorphism (RFLP) (Genequality 12-13 K-RAS, IVD marked, AB ANALITICA, Padua, Italy) and pyrosequencing (PyroMark THQ96-QIAgen, Germany), with Anti-EGFR MoAb response-KRAS status (Diatech Pharmacogenetics, Jesi, Italy).

REALQUALITY RI-KRAS MuST kit is an in vitro test for the detection and typing of the seven most frequent mutations in the KRAS oncogene; it includes all reagents ready to use and positive controls necessary for the execution of the test. The kit is based on a mutation specific amplification of DNA from FFPE by Real-Time SSP-PCR (PCR-SequenceSpecific Primers): when the primer is fully matched, the amplification proceeds with full efficiency; when the primer is mismatched at the $3^{\prime}$ bases, only low amplification occurs. The detection of specific amplification is performed by using a DNA-binding dye that is able to intercalate in the DNA molecules without inhibiting the PCR reaction and is both extremely thermally and hydrolytically stable. Moreover, being completely impermeable to cells membranes, it is not mutagenic or cytotoxic. The use of this DNA-binding dye allows melting curve analysis to determine the characteristic melting temperature (Tm) of the target DNA and to identify or genotype PCR products based on their Tm. The amplicon length for both control gene and mutation is about $150 \mathrm{bp}$, an optimal length for amplification efficiency in Real-Time PCR and for heavily fragmented FFPE samples.

The method has been validated using 3 different kit lots on Applied Biosystems 7500 Fast DX, with software version 1.4 (Applied Biosystems). The analytical sensitivity was evaluated by dissociation curve analysis on mixtures of mutated DNA (pancreatic cancer cell line CAPAN-1 for G12V mutation, plasmid DNA for other mutation), corresponding to $5 \%, 2.5 \%, 1 \%, 0.5 \%$, and $0 \%$, in a background of KRAS wild-type DNA (human colonic adenocarcinoma cell line T-84), over a range of three different concentrations of background DNA from about $70 \mathrm{ng} /$ reaction to $5 \mathrm{ng} /$ reaction and repeated in three consecutive sessions. The use of different concentration of background DNA 


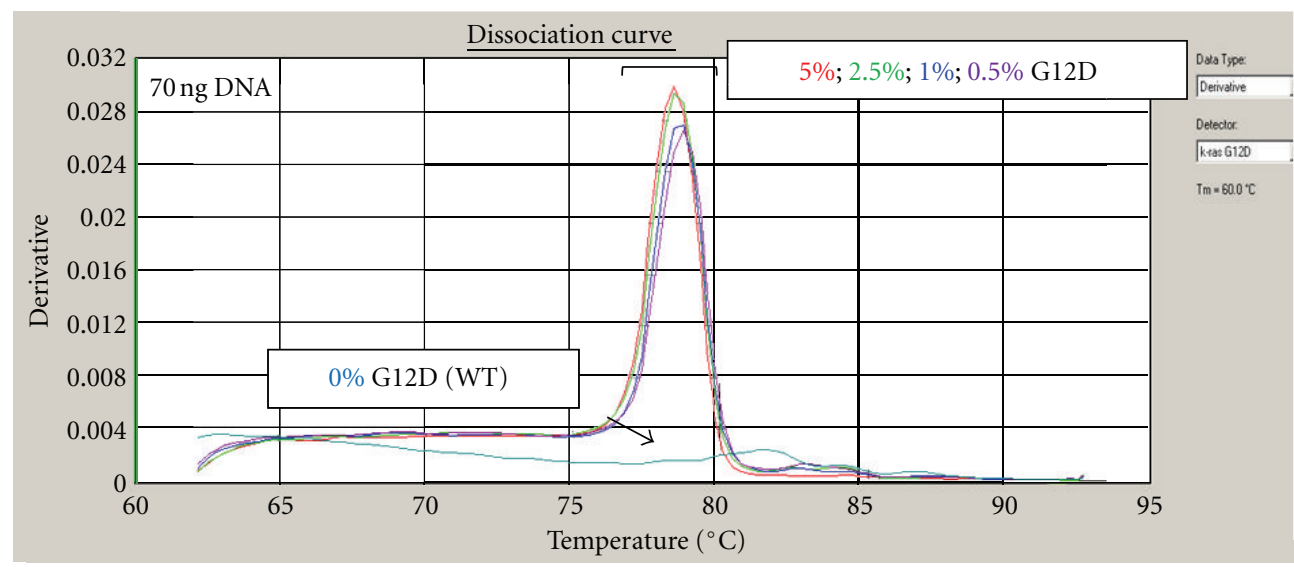

(a)

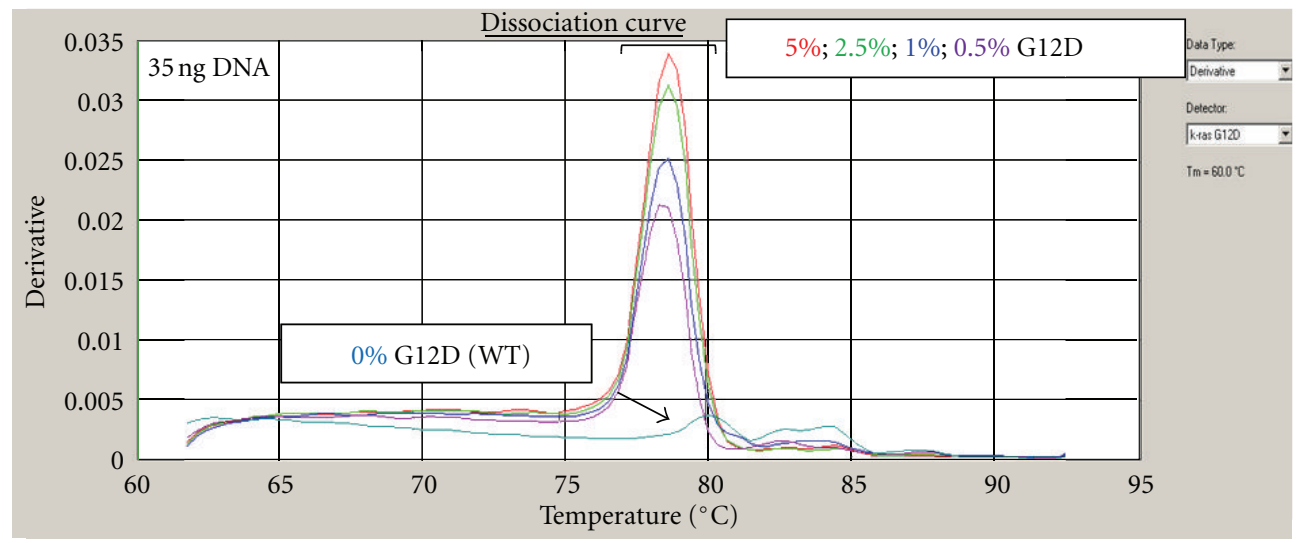

(b)

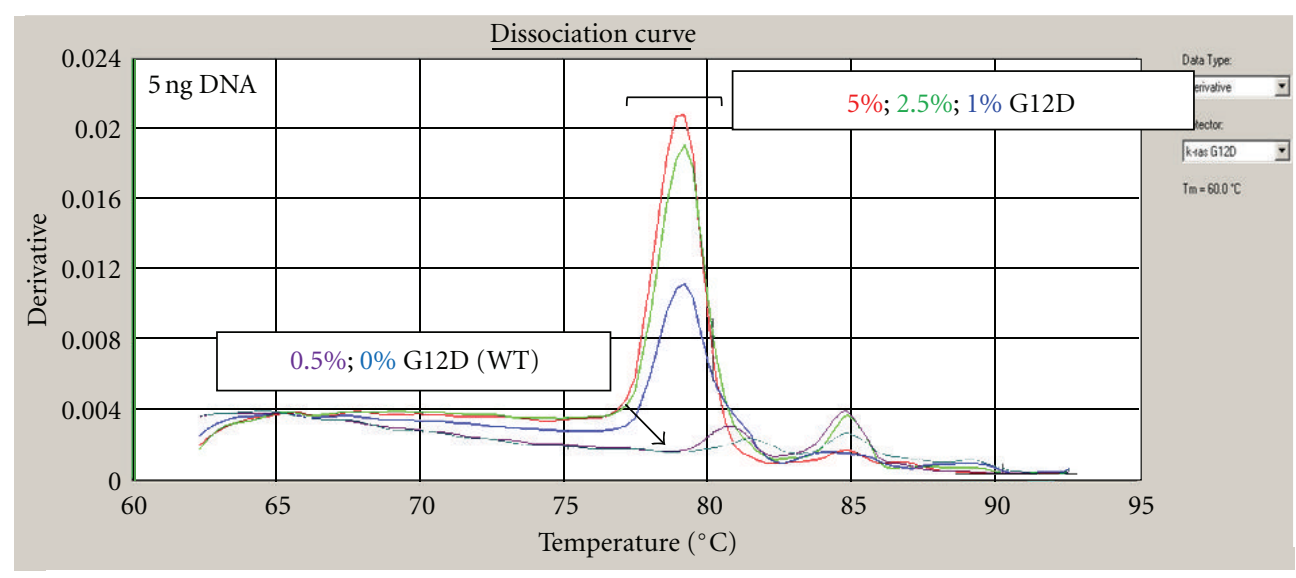

(c)

FIGURE 1: Dissociation curves from KRAS analytical sensitivity experiment for G12D KRAS mutation. The analysis corresponds to 5\%, $2.5 \%, 1 \%, 0.5 \%$, and $0 \%$ G12D in different amounts of DNA background from T-84 cell line: for background DNA of 70 ng/reaction (a) and $35 \mathrm{ng} /$ reaction (b) the PCR products of 5\%, 2.5\%, 1\%, 0.5\% mutated DNA show specific melting curve until 0.5\%. For background DNA of $5 \mathrm{ng} /$ reaction (c) the specific dissociation curve is present until $1 \%$.

was to mimic as closely as possible the nature of clinical sample. REALQUALITY RI-KRAS MuST showed to identify unambiguously the KRAS mutation in mixtures containing at least 1\% mutant DNA, for all DNA background (Figure 1).

Since the use of cell lines or plasmid DNA does not reflect the clinical setting of tumour samples, leading to over- interpretation of the results, the cut-off values for each assay mutation has not been determined from analytical sensitivity experiments but from analysis of 182 FFPE samples. Clinical specimens tend to show worse levels of breakthrough, that is defined as the nonspecific amplification of a wild-type DNA sample; therefore, we chose to set the cut-off as the difference 
TABLE 1: Summary of the KRAS mutational status.

\begin{tabular}{lcc}
\hline & $\begin{array}{c}\text { Direct } \\
\text { sequencing }\end{array}$ & $\begin{array}{c}\text { REALQUALITY } \\
\text { RI-KRAS MuST }\end{array}$ \\
\hline Number of samples analyzed & 182 & 182 \\
Wild-type status & 115 & 106 \\
Mutated & 59 & $* 76(59+17)$ \\
Weak or unclear mutation & 8 & 0 \\
\hline
\end{tabular}

Direct Sequencing (DS) versus REALQUALITY RI-KRAS MuST (RQ), * 76 cases $=59$ mutated cases by SD +17 mutated cases by RQ but not by SD.

between the cycle threshold $(\mathrm{Ct})$ from mutation reaction and the $\mathrm{Ct}$ from control reaction $(\Delta \mathrm{Ct})$ value corresponding to the maximum diagnostic sensitivity and specificity.

\section{Results and Discussion}

This study analyzed 182 patients. Direct sequencing identified KRAS wild type in 115 cases and mutations in exon 2 in 59 cases (Table 1$)(n=17$ G12D, $n=15 \mathrm{G} 12 \mathrm{~V}, n=15$ G13D, $n=5$ G12A, $n=4$ G12C, $n=2$ G12S, $n=1$ G12R). In other 8 cases the result was weak or unclear mutation.

REALQUALITY RI-KRAS MUST confirmed all the mutated cases (59); in addition, it detected KRAS mutations in other 17 cases, 9 of which resulted negative and 8 weak/ ambiguous (low quality of sequence) at direct sequencing (Table 1). This subgroup of 17 samples were additionally analyzed by pyrosequencing and RFLP-PCR (Table 2). A cohort of 4 cases showed a mutation in exon $2(n=2$ G12A; $n=1 \mathrm{G} 12 \mathrm{~V}, n=1 \mathrm{G} 12 \mathrm{C}$ ) detectable with the REALQUALITY RI-KRAS MuST and pyrosequencing, but not by RFLP-PCR. These data confirm the higher sensitivity of REALQUALITY RI-KRAS MuST (sensitivity 1\%: mutant/wild type) compared with direct sequencing (sensitivity 20\%-mutant/wild type) and RFLP-PCR (sensitivity 10\%-mutant/wild type) [24-27]. Although all the analyzed samples were selected by macrodissection, the direct sequencing did not detect mutations in 17 samples because of the genetic heterogeneity of the biopsy material. In addition, the amount of tumor versus nontumor area results in a template mixture in which wild-type and mutant DNAs are not present in equimolar amounts, and we have to consider also that there are differences in the detection limits for distinct mutations [26].

In the REALQUALITY RI-KRAS MuST data analysis utilizes the number of PCR cycles required to detect a fluorescent signal above a background signal ( $\mathrm{Ct}-$ cycle threshold) as a measurement of the specific target molecules present in the sample at the beginning of the reaction. Furthermore, the melting curve analysis of PCR product helps to define the specificity of the reaction, since, when using sequencespecific primers, some nonspecific priming may occur giving a late $\mathrm{Ct}$ from sample not containing mutation (Figure 2).

Sample $\Delta \mathrm{Ct}$ values are calculated as the difference between the $\mathrm{Ct}$ taken from mutation reaction and the $\mathrm{Ct}$ given by the control reaction. As result, the amplification of a control gene is required for both assessment of total DNA in the sample and classification as mutation positive or negative. Specifically, for a certain mutation, if the sample produces a Tm of PCR product included in the specific range and a $\Delta \mathrm{Ct}$ less than the cut-off value, the sample is classified as mutation positive. The cut-off value is the smaller $\Delta \mathrm{Ct}$ with the corresponding maximum of diagnostic sensitivity and specificity. The diagnostic sensitivity, that is, the number of positive samples on the total number of true positive, was $100 \%(59 / 59)$. The diagnostic specificity, that is, the number of negative samples on the total number of true negative, hit $100 \%$ of the cases $(106 / 106)$. Based on the results obtained (Tables 1 and 2) we can then calculate the recovery rate of the REALQUALITY RI-KRAS MuST as $17 /(106+$ 17) corresponding to $13,82 \%$ of recovered positive samples among 123 negative samples analyzed by direct sequencing.

\section{Conclusions}

CRC is one of the major causes of cancer-related mortality. The EGFR signaling pathway is frequently activated in CRC and has been extensively investigated as a target for cancer therapy. During the American Society of Clinical Oncology meeting (ASCO) in 2008, evidence was presented in which patients with CRC in advanced stages would not receive any benefit from the administration of cetuximab (Erbitux) and panitumumab (Vectibix), if there were mutations in the KRAS gene. Therefore, the KRAS mutational state is a predictive marker for anti-EGFR therapy. The Food and Drug Administration and the European Medicines Agency have approved the analysis of KRAS mutation status as a diagnostic tool to select metastatic CRC patients eligible to be treated with cetuximab or panitumumab. Several methodological variables can affect the outcome of testing for KRAS mutations in formalin-fixed, paraffin-embedded samples [28].

Currently, the most widely applied method for assessing KRAS gene status is direct sequencing of PCR amplification products. This method has a relatively low sensitivity because mutant alleles must be present in at least $20 \%$ of cells to be reproducibly detected $[3,29,30]$.

In this perspective, the new REALQUALITY RI-KRAS MuST is a more sensitive method for KRAS analysis. In fact, the analytical sensitivity was at least $1 \%$ mutant DNA, the diagnostic sensitivity and specificity was $100 \%$, and the recovery rate of positive samples versus direct sequencing was $13,82 \%$. The main advantage of REALQUALITY RIKRAS MuST compared to other Real-Time PCR systems for KRAS mutation analysis is the use of a next-generation DNA binding instead of a fluorescent probe $[4,31]$. This approach allows to perform the post-PCR melting curve analysis and then verify the reaction specificity. Therefore, the correctness of the result is based not only on the difference between $\mathrm{Ct}$ from mutation reaction and from control reaction $(\Delta \mathrm{Ct})$ but also on Tm analysis of PCR product that is an index of specificity. Despite the large number of reactions to be performed (one for control gene and one for each of the 7 KRAS mutations) and the need to complete the kit with a software for the automatic interpretation of results, the high accuracy of REALQUALITY RI-KRAS MuST is clinically relevant and suggests a possible extensive use in the laboratory of surgical pathology. 


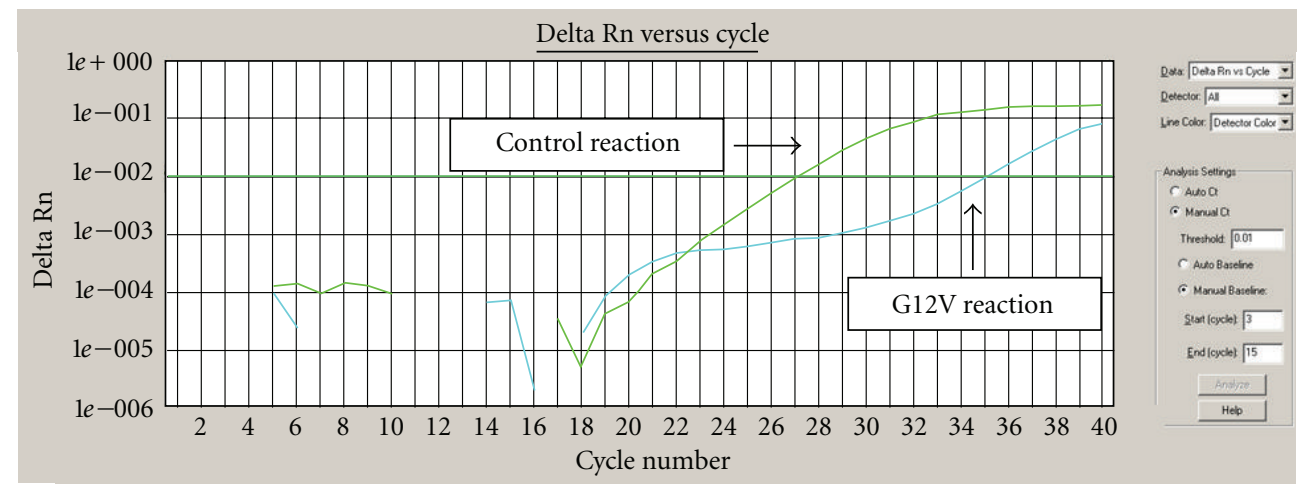

(a)

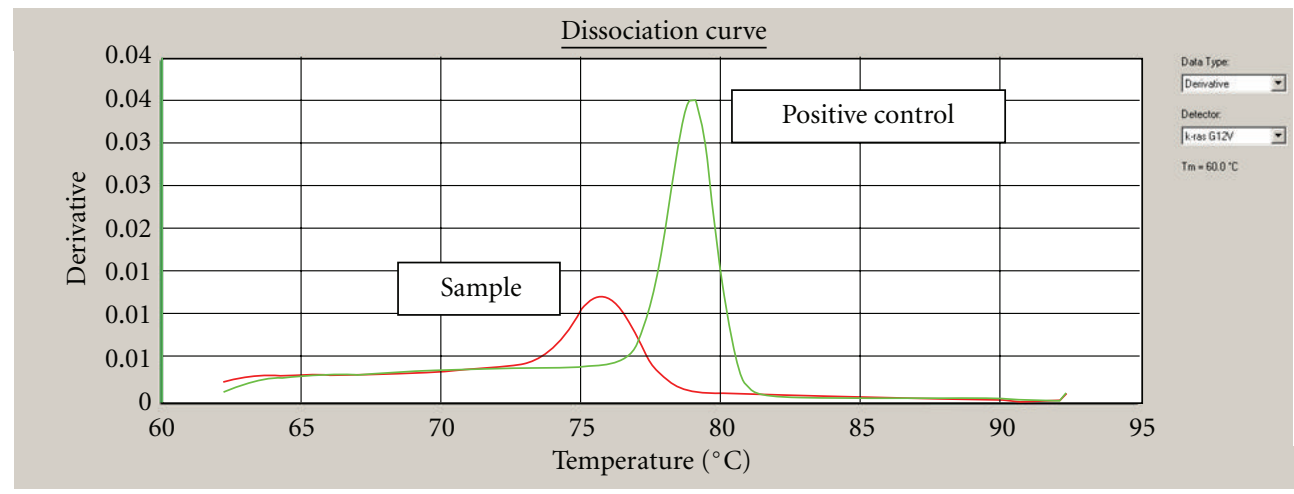

(b)

FIGURE 2: Example of nonspecific priming and breakthrough level analysis of KRAS amplification: the sample produces an amplification curve for both control gene and G12V reaction, even though it is wild type (a), but the melting curve of sample compared with positive control (provided in REALQUALITY RI-KRAS MuST) shows a different Tm (b). The sample can therefore be classified as wild type for G12V mutation.

TABLE 2: Results of KRAS mutation testing by different methodologies.

\begin{tabular}{|c|c|c|c|c|}
\hline Sample ID & Direct sequencing & REALQUALITY RI-KRAS MuST & RFLP-PCR & Pyrosequencing \\
\hline 017UD & Weak G12V & $\mathrm{G} 12 \mathrm{~V}$ & Mutated codon 12 & G12V \\
\hline 076UD & *Unclear sequence G12V & G12V & Mutated codon 12 & G12V \\
\hline 088UD & *Unclear sequence G12V & G12V & Mutated codon 12 & G12V \\
\hline 044UD & $\begin{array}{l}\text { First sequence: wild-type Repeat } \\
\text { sequence: }{ }^{*} \text { Unclear G12V }\end{array}$ & G12V & Mutated codon 12 & G12V \\
\hline 047UD & $\begin{array}{l}\text { First sequence: wild-type Repeat } \\
\text { sequence: *Unclear G12V }\end{array}$ & G12V & Mutated codon 12 & G12V \\
\hline 120UD & $\begin{array}{l}\text { First sequence: weak G12D Repeat } \\
\text { sequence: wild type }\end{array}$ & G12D & Mutated codon 12 & G12D \\
\hline 082UD & *Unclear sequence G13D & G13D & Mutated codon 13 & G13D \\
\hline 068UD & $\begin{array}{c}\text { First sequence: wild-type Repeat } \\
\text { sequence: weak G13D }\end{array}$ & G13D & Mutated codon 13 & G13D \\
\hline 036UD & Wild type & G12D & Mutated codon 12 & G12D \\
\hline 046UD & Wild type & G12D & Mutated codon 12 & G12D \\
\hline 077UD & Wild type & $\mathrm{G} 12 \mathrm{~V}$ & Mutated codon 12 & G12V \\
\hline 106UD & Wild type & G12C & Mutated codon 12 & G12C \\
\hline 059UD & Wild type & G12S & Mutated codon 12 & G12S \\
\hline 015UD & Wild type & G12A & Negative & G12A \\
\hline 145UD & Wild type & G12C & Negative & G12C \\
\hline 041UD & Wild type & G12V & Negative & G12V \\
\hline 043UD & Wild type & G12A & Negative & G12A \\
\hline
\end{tabular}

Abbreviations. Direct Sequencing: DS; REALQUALITY RI-KRAS MuST: RQ; pyrosequencing: PS. * Unclear sequence $=$ low quality of sequence. 
In conclusion, there is a general consensus that seven mutations in codons 12 and 13 of the KRAS gene should be covered, but other mutations need to be analysed, such as the BRAF gene mutation that is present in about $60 \%$ of nonresponder patients with KRAS wild type. The next goal is just to develop a similar kit for the determination of the mutation in the BRAF V600E gene.

\section{Authors' Contribution}

S. Marzinotto and F. Sessa are contributed equally to this work.

\section{References}

[1] F. Di Fiore, F. Blanchard, F. Charbonnier et al., "Clinical relevance of KRAS mutation detection in metastatic colorectal cancer treated by Cetuximab plus chemotherapy," British Journal of Cancer, vol. 96, no. 8, pp. 1166-1169, 2007.

[2] F. Molinari, L. Felicioni, M. Buscarino et al., "Increased detection sensitivity for KRAS mutations enhances the prediction of anti-EGFR monoclonal antibody resistance in metastatic colorectal cancer," Clinical Cancer Research, vol. 17, no. 14, pp. 4901-4914, 2011.

[3] A. Sartore-Bianchi, K. Bencardino, A. Cassingena et al., "Therapeutic implications of resistance to molecular therapies in metastatic colorectal cancer," Cancer Treatment Reviews, vol. 36, no. 3, pp. S1-S5, 2010.

[4] J. H. J. M. Van Krieken, A. Jung, T. Kirchner et al., "KRAS mutation testing for predicting response to anti-EGFR therapy for colorectal carcinoma: proposal for an European quality assurance program," Virchows Archiv, vol. 453, no. 5, pp. 417431, 2008.

[5] B. George and S. Kopetz, "Predictive and prognostic markers in colorectal cancer," Current Oncology Reports, vol. 13, no. 3, pp. 206-215, 2011.

[6] D. L. Worthley, V. L. Whitehall, K. J. Spring, and B. A. Leggett, "Colorectal carcinogenesis: road maps to cancer," World Journal of Gastroenterology, vol. 13, no. 28, pp. 37843791, 2007.

[7] C. S. Karapetis, S. Khambata-Ford, D. J. Jonker et al., "K-ras mutations and benefit from cetuximab in advanced colorectal cancer," New England Journal of Medicine, vol. 359, no. 17, pp. 1757-1765, 2008.

[8] F. Kabbinavar, H. I. Hurwitz, L. Fehrenbacher et al., "Phase II, randomized trial comparing bevacizumab plus fluorouracil (FU)/leucovorin (LV) with FU/LV alone in patients with metastatic colorectal cancer," Journal of Clinical Oncology, vol. 21, no. 1, pp. 60-65, 2003.

[9] H. Hurwitz, L. Fehrenbacher, W. Novotny et al., "Bevacizumab plus irinotecan, fluorouracil, and leucovorin for metastatic colorectal cancer," New England Journal of Medicine, vol. 350, no. 23, pp. 2335-2342, 2004.

[10] L. B. Saltz, N. J. Meropol, P. J. Loehrer, M. N. Needle, J. Kopit, and R. J. Mayer, "Phase II trial of cetuximab in patients with refractory colorectal cancer that expresses the epidermal growth factor receptor," Journal of Clinical Oncology, vol. 22, no. 7, pp. 1201-1208, 2004.

[11] R. G. Amado, M. Wolf, M. Peeters et al., "Wild-type KRAS is required for panitumumab efficacy in patients with metastatic colorectal cancer," Journal of Clinical Oncology, vol. 26, no. 10, pp. 1626-1634, 2008.
[12] E. van Cutsem, C. H. Köhne, E. Hitre et al., "Cetuximab and chemotherapy as initial treatment for metastatic colorectal cancer," New England Journal of Medicine, vol. 360, no. 14, pp. 1408-1417, 2009.

[13] A. Lièvre, H. Blons, and P. Laurent-Puig, "Oncogenic mutations as predictive factors in colorectal cancer," Oncogene, vol. 29, no. 21, pp. 3033-3043, 2010.

[14] D. Cunningham, Y. Humblet, S. Siena et al., "Cetuximab monotherapy and cetuximab plus irinotecan in irinotecanrefractory metastatic colorectal cancer," New England Journal of Medicine, vol. 351, no. 4, pp. 337-345, 2004.

[15] G. Schuch, S. Kobold, and C. Bokemeyer, "Evolving role of cetuximab in the treatment of colorectal cancer," Journal of Cancer Management and Research, vol. 1, pp. 79-88, 2009.

[16] G. Tonini, A. Calvieri, B. Vincenzi, and D. Santini, "First-line targeted therapies in the treatment of metastatic colorectal cancer - Role of cetuximab," OncoTargets and Therapy, vol. 2, pp. 73-82, 2009.

[17] A. Sartore-Bianchi, F. Di Nicolantonio, M. Nichelatti et al., "Multi-determinants analysis of molecular alterations for predicting clinical benefit to EGFR-targeted monoclonal antibodies in colorectal cancer," PLoS ONE, vol. 4, no. 10, Article ID e7287, 2009.

[18] R. Reddavide, G. Misciagna, M. G. Caruso et al., "Tissue expression of glycated apolipoprotein B in colorectal adenoma and cancer," Anticancer Research, vol. 31, no. 2, pp. 555-559, 2011.

[19] D. L. Wheeler, E. F. Dunn, and P. M. Harari, "Understanding resistance to EGFR inhibitors-impact on future treatment strategies," Nature Reviews Clinical Oncology, vol. 7, no. 9, pp. 493-507, 2010.

[20] A. Bardelli and S. Siena, "Molecular mechanisms of resistance to cetuximab and panitumumab in colorectal cancer," Journal of Clinical Oncology, vol. 28, no. 7, pp. 1254-1261, 2010.

[21] M. Frattini, G. Gallino, S. Signoroni et al., "Quantitative analysis of plasma DNA in colorectal cancer patients: a novel prognostic tool," Annals of the New York Academy of Sciences, vol. 1075, pp. 185-190, 2006.

[22] B. Angulo, E. García-García, R. Martínez et al., "A commercial real-time PCR kit provides greater sensitivity than direct sequencing to detect KRAS mutations: a morphologybased approach in colorectal carcinoma," Journal of Molecular Diagnostics, vol. 12, no. 3, pp. 292-299, 2010.

[23] W. De Roock, B. Claes, D. Bernasconi et al., "Effects of KRAS, BRAF, NRAS, and PIK3CA mutations on the efficacy of cetuximab plus chemotherapy in chemotherapy-refractory metastatic colorectal cancer: a retrospective consortium analysis," The Lancet Oncology, vol. 11, no. 8, pp. 753-762, 2010.

[24] S. M. Anderson, "Laboratory methods for KRAS mutation analysis," Expert Review of Molecular Diagnostics, vol. 11, no. 6, pp. 635-642, 2011.

[25] A. C. Tsiatis, A. Norris-Kirby, R. G. Rich et al., "Comparison of Sanger sequencing, pyrosequencing, and melting curve analysis for the detection of KRAS mutations: diagnostic and clinical implications," Journal of Molecular Diagnostics, vol. 12, no. 4, pp. 425-432, 2010.

[26] Z. Zuo, S. S. Chen, P. K. Chandra et al., "Application of COLDPCR for improved detection of KRAS mutations in clinical samples," Modern Pathology, vol. 22, no. 8, pp. 1023-1031, 2009.

[27] P. Nollau and C. Wagener, "Methods for detection of point mutations: performance and quality assessment. The IFCC Scientific Division, Committee on Molecular Biology Techniques," Journal of the International Federation of Clinical Chemistry, vol. 9, no. 4, pp. 162-170, 1997. 
[28] J. H. J. M. van Krieken, A. Jung, T. Kirchner et al., "KRAS mutation testing for predicting response to anti-EGFR therapy for colorectal carcinoma: proposal for an European quality assurance program," Virchows Archiv, vol. 453, no. 5, pp. 417431, 2008.

[29] W. A. Franklin, J. Haney, M. Sugita, L. Bemis, A. Jimeno, and W. A. Messersmith, "KRAS mutation: comparison of testing methods and tissue sampling techniques in colon cancer," Journal of Molecular Diagnostics, vol. 12, no. 1, pp. 43-50, 2010.

[30] A. Jimeno, W. A. Messersmith, F. R. Hirsch, W. A. Franklin, and S. G. Eckhardt, "KRAS mutations and susceptibility to cetuximab and panitumumab in colorectal cancer," Cancer Journal, vol. 15, no. 2, pp. 110-113, 2009.

[31] N. Thelwell, S. Millington, A. Solinas, J. Booth, and T. Brown, "Mode of action and application of Scorpion primers to mutataon detection," Nucleic Acids Research, vol. 28, no. 19, pp. 3752-3761, 2000. 


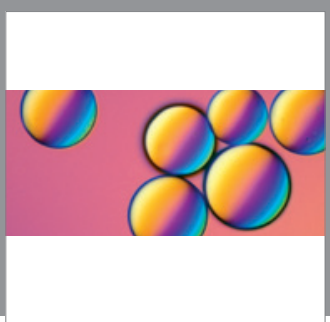

Peptides

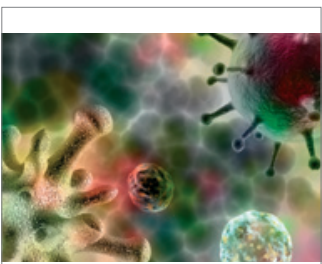

\section{Virology}

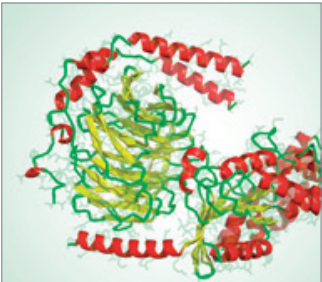

Signal Transduction
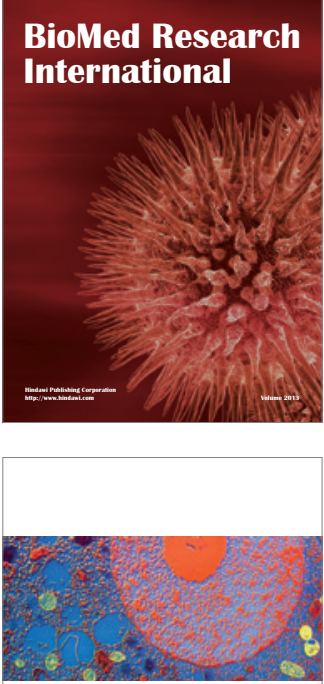

ISRN

Cell Biology
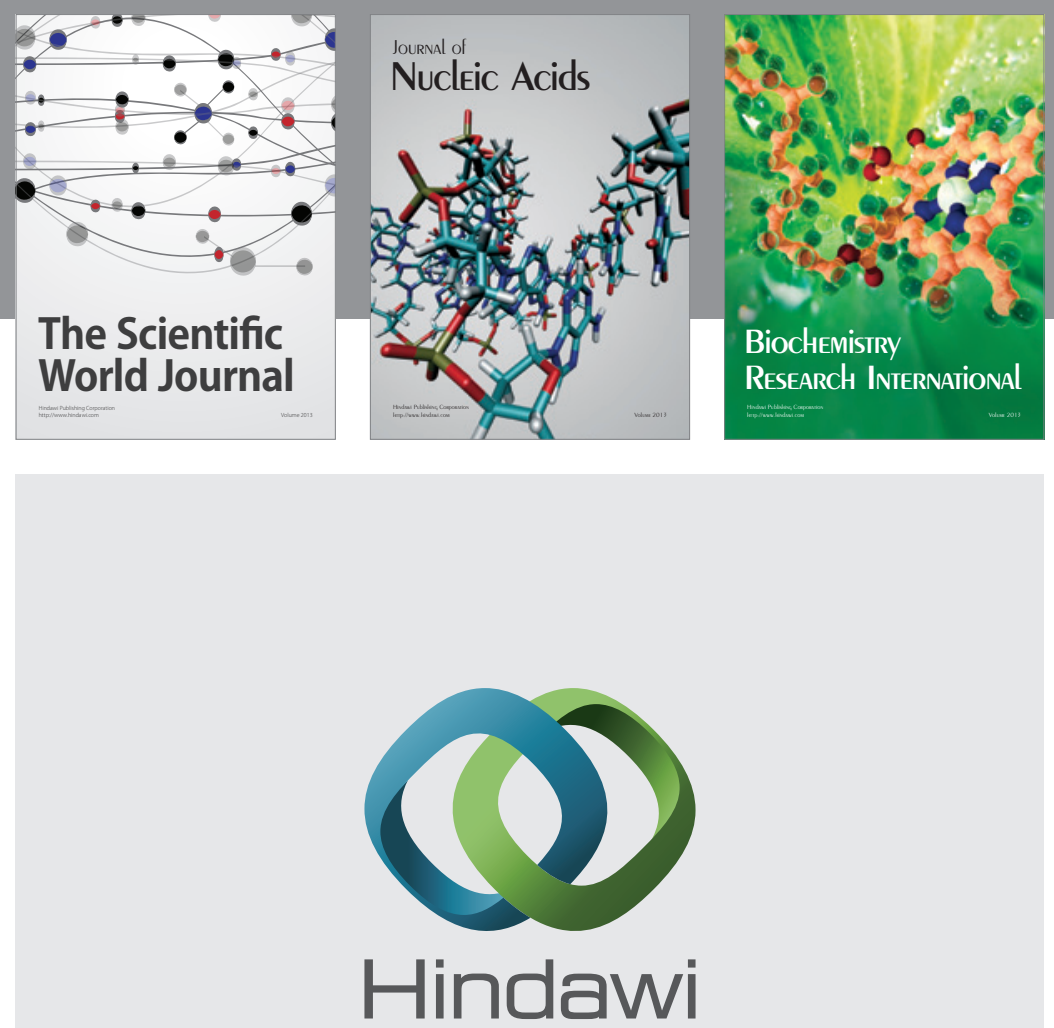

Submit your manuscripts at http://www.hindawi.com

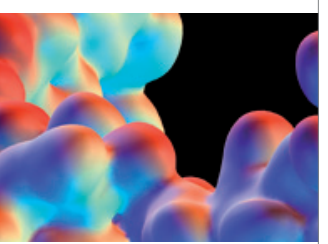

Enzyme Research

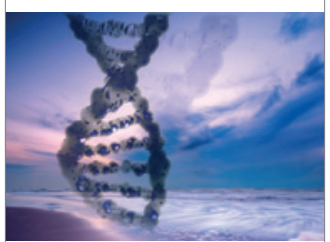

Evolutionary Biology
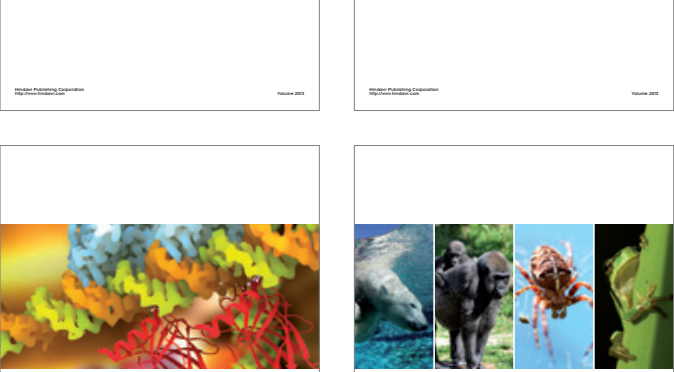

ISRN

Molecular Biology

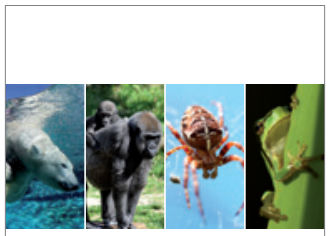

ISRN Zoology

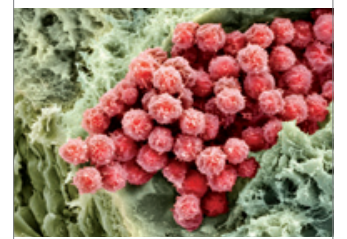

Stem Cells International

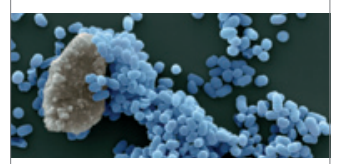

ISRN

Biotechnology
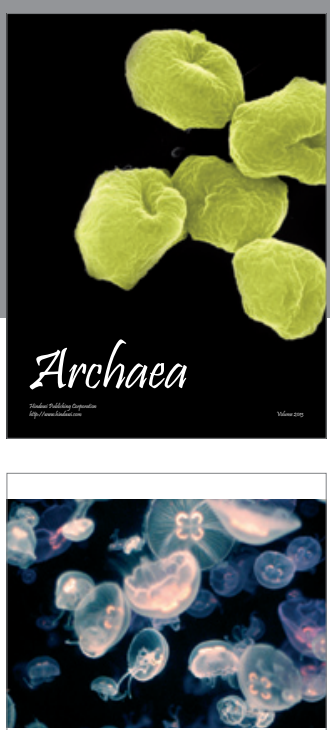

Journal of Marine Biology
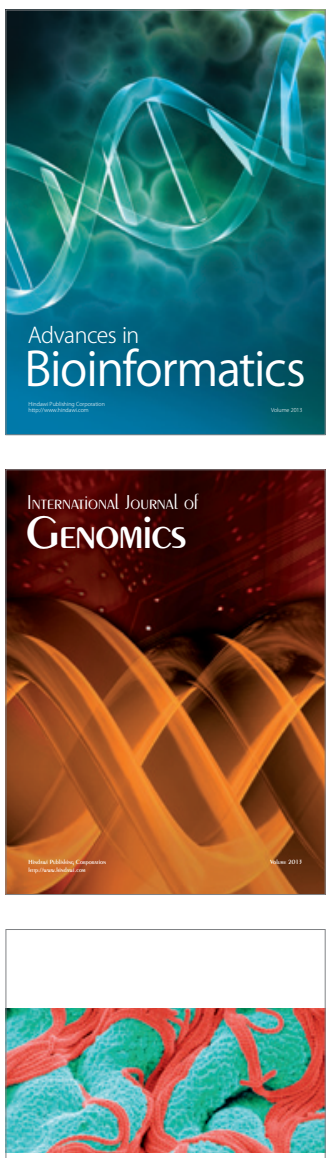

ISRN

Microbiology 